\title{
Sedation for dental treatment of children in the primary care sector (UK)
}

\author{
P. F. Ashley, ${ }_{1}$ J. Parry, ${ }^{2}$ S. Parekh, ${ }^{3}$ M. Al-Chihabi ${ }^{4}$ and D. Ryan ${ }^{5}$
}

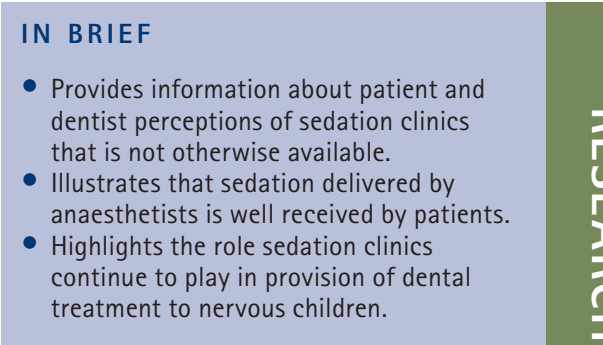

\begin{abstract}
Objective To audit the clinical practice of a dental sedation service in the primary care sector and determine which services dentists use to manage unco-operative children. Design Retrospective analysis and prospective audit. Setting Sedation clinic in primary care, 2007, England. Subjects Children attending for dental treatment under sedation. General dental practitioners (GDPs) in the Brighton and West Sussex regions. Interventions Questionnaire. Main outcome measures Clinical service audit, patient satisfaction, referrer satisfaction. Results Four hundred children (age range 5-12 years) had been referred for caries (78\%), with the remainder for orthodontic extractions. The most common treatment carried out on primary and permanent teeth was extractions followed by restorations. A combination of intravenous (IV) midazolam/ketamine/fentanyl was used in 40\% of cases, and IV midazolam/ketamine was used in 34\% of cases. Seventy-four percent of parents responded to the satisfaction questionnaire; of these $97 \%$ rated sedation as excellent/good and $80 \%$ would choose sedation or recommend sedation for others. Only $45 \%$ of questionnaires to referrers were returned. Fifty-six percent of dentists preferred general anaesthesia (GA) and 66\% preferred IV sedation. Conclusions Dental treatment for children was provided under IV sedation with most parents satisfied with the procedure. Little difference was seen between referring dentists' perceptions of IV sedation or GA.
\end{abstract}

\section{INTRODUCTION}

It is well recognised that providing dental care for children can be problematic. Reasons for this include fear of pain, anxiety or behaviour management problems (BMP). Therefore methods to manage BMP or dental fear are required. Non-pharmacological behaviour management techniques have an important role but are not the solution for all children; a proportion will require a pharmacological approach such as general anaesthesia (GA) or sedation.

In the United Kingdom (UK), GA is commonly used for the provision of dental

\footnotetext{
${ }^{1 *}$ Senior Lecturer, Honorary Consultant and Head of Unit, ${ }^{3}$ Clinical Lecturer and Programme Coordinator, Department of Paediatric Dentistry, UCL Eastman Dental Institute, 256 Gray's Inn Road, London, WC1X 8LD; ${ }^{2}$ West Sussex Primary Care Trust, Haywards Heath Health Centre, Heath Road, Haywards Heath, RH16 3BB ${ }^{4}$ Ulster Hospital, Upper Newtownards Road, Dundonald, Belfast, Co Down, BT16 1RH; ${ }^{5}$ General Dental Practitioner, St Faith Dental Clinic, 2 Halsford Park Road, East Grinstead, West Sussex, RH19 1PN

*Correspondence to: Dr Paul Ashley

Email: p.ashley@eastman.ucl.ac.uk
}

Online article number E21

Refereed Paper - accepted 24 April 2010

DOI: 10.1038/sj.bdj.2010.542

${ }^{\circledR}$ British Dental Journal 2010: E21 treatment in children. Up until 2000, a large proportion of these GAs were carried out in the primary care sector, however a change in regulations ${ }^{1}$ required that general anaesthesia for dentistry be restricted to a hospital setting. Since the implementation of this legislation, many former primary care providers of dental general anaesthetics (DGAs) now provide a similar dental service for children using polypharmaceutical intravenous (IV) sedation provided by anaesthetists.

Use of standard sedation techniques in children is limited at present to nitrous oxide/oxygen $\left(\mathrm{N}_{2} \mathrm{O} / \mathrm{O}_{2}\right)$ in the UK. The use of poly-pharmaceutical sedation for dental treatment in children generates significant debate whether administered by a dentist or anaesthetist. ${ }^{2}$ It is therefore unfortunate that little or no information exists describing existing services provided in the UK by these clinics for children.

Therefore the aims of this project were:

1. To audit the clinical practice of a dental sedation service in the primary care sector

2. To determine which services dentists use to manage unco-operative children (sedation or general anaesthesia).

\section{MATERIALS AND METHODS}

This paper presents patient data collected from audits carried out with the co-operation of a sedation clinic based in the South-East of England. The clinic lies in a convenient location to serve patients living in Kent, Surrey and Sussex. This clinic provides dental care for children and adults under intravenous sedation. Patients are mainly referred from the surrounding area and the sedation is provided by anaesthetists. General anaesthetic facilities are not available in the primary care sector. A sample of GDP contact details was provided by West Sussex and Brighton and Hove primary care trusts (PCTs) in order to audit GDPs in those areas with regard to service satisfaction.

\section{Audit of clinical practice of a dental sedation service in the primary care sector}

This was carried out in collaboration with a sedation clinic located in the South-East of England. 
Data was extracted from patient records between 2002 and 2005. One hundred notes were sampled from each year (MA-C selected notes from different points in the storage area), resulting in a total of 400 patient notes. The audit inclusion criteria were:

- Patients under 16 years of age at the time of the procedure

- All data required available from the records.

A pilot form was devised, and then adapted after testing in a sample of five patient notes. A list of information of interest was developed; this was then matched to data available. The information finally recorded is shown in Table 1.

\section{Patient satisfaction}

A questionnaire to measure satisfaction was adapted and modified from a previous paper. ${ }^{3}$ Key points of the questionnaire are shown in Table 2. Any patients who were receiving dental treatment from the sedation clinic and were under 16 years of age were included.

These questionnaires were given immediately on completion of the dental procedure to the parent/carer by the operator. They were asked to fill out the questions 24 hours post- treatment and to return the questionnaire in a prepaid envelope. One hundred envelopes were given to the first 100 parents/carers attending the clinic with children less than 16 years of age requiring treatment.

\section{Dentists' preferences between general anaesthesia and intravenous sedation}

All registered dentists were targeted in Brighton and Hove (64 registered dentists) and West Sussex (157 registered dentists) PCTs. A total of 221 questionnaires were sent (64 to Brighton and 157 to West Sussex). This part of the study was carried out as an audit in collaboration with the Brighton and Hove and West Sussex PCTs.

The questionnaire was developed from a previous study carried out in the UK; ${ }^{4}$ details are shown in Table 3. The addresses of the dentists were provided by the relevant PCT. The questionnaires recorded demographic details of the dentist and then assessed their satisfaction with available GA or IV sedation services for children. The questionnaire is illustrated in Table 3.

\section{Statistical analysis}

Data were entered into a database, checked for errors, and analysed with the use of SPSS-12 software (SPSS Inc, Illinois, USA) and simple descriptive statistics.

\section{RESULTS}

\section{Audit of clinical practice of a dental sedation service in the primary care sector}

Four hundred children, 202 male and 198 female, with a mean age of 8.5 years (range 5-12 years) had all been referred from their general dental practitioners (GDPs). Most of the children were in good health (81\%), while the rest reported conditions such as asthma (10\%) or allergy to antibiotic (5\%).

The majority of children were referred for caries $(312(78 \%))$, with the remainder for orthodontic extractions. The main types of treatment provided are shown in Figure 1 with the most common treatment carried out on primary and permanent teeth being extractions, followed by restorations. Children having extractions in the primary dentition had on average 2.4 teeth extracted (SD 1.6, range 1-10) and children having extractions in the permanent dentition had on average 2.7 teeth extracted (SD 1.2, range 1-4). Children having restorations of the primary dentition had on average 3 teeth restored (SD 1.6, range

\begin{tabular}{l} 
Table 1 Information recorded in the \\
audit of clinical practice in dental \\
sedation service \\
Year of treatment \\
\hline Patient demographics \\
\hline Name \\
\hline Gender \\
\hline Date of birth \\
\hline Medical history \\
\hline Referral details \\
\hline Practitioner responsible for referral \\
\hline Reason for referral \\
\hline Types of treatment carried out \\
\hline Sedation procedure \\
\hline Patients' weight \\
\hline Agent used and dose \\
\hline Adverse reactions \\
\hline Number of visits required for treatment \\
\hline
\end{tabular}

\section{Table 2 Key points of the patient satisfaction questionnaire}

Question 1 enquired about the patient's experience with the dental treatment under sedation.

Questions 2 to 4 enquired about post-operative complaints after 1 hour, 6 hours and 24 hours. Responses included pain, bleeding, dizziness, sleepiness, weakness, and others.

Questions 5 and 6 asked the parents if they would recommend the service to other children, and if they would consider repeating treatment for their children under intravenous sedation.

\section{Table 3 Dentists' perceptions of GA versus sedation}

Demographic details - gender and year of qualification

The following questions were asked on separate sheets of paper for both GA and IV sedation:

Reasons for referral:

- Patient fails to respond to other form of behaviour management

- Too young to cooperate

- Complicated Treatment

- Parents Request

- Get treatment completed in one visit

- Special needs patient.

Age group of patients you most often refer?

Where do you normally refer the patient?

Normal waiting time before the patient received treatment

Number of children referred per month on average

Satisfaction with the service (scale from 1 to 5 ) 
1-9) and children having restorations of the permanent dentition had on average 2.6 teeth restored (SD 1.4, range 1-8).

Local anaesthetic (LA) was only used in 180 patients (45\%). No adverse events were recorded.

The number of visits to complete the treatment was one for $96 \%$ of children and two for 3\%; only two patients needed three visits to complete the treatment (1\%).

A combination of IV midazolam/ketamine/fentanyl was used in 40\% of cases and IV midazolam/ketamine was used in $34 \%$ of cases. The frequency of combinations used is summarised in Figure 2. The midazolam doses ranged between 0.2-8 $\mathrm{mg}$ with $2 \mathrm{mg}(32 \%)$ and $3 \mathrm{mg}(16 \%)$ the most common doses, while the ketamine doses ranged between $1-50 \mathrm{mg}$ with $10 \mathrm{mg}$ the most common dose (24\%). Fentanyl doses ranged between 7.5-50 $\mu \mathrm{g}$ with $25 \mu \mathrm{g}$ the most common dose (26\%).

\section{Patient satisfaction}

A total of 100 questionnaires were given out to parents. Only 58 responded, so reminders were sent after one month. Sixteen more were then returned. In total we received 74 replies from parents.

Of the parents who responded, 72 (97\%) rated sedation as excellent or good. Fiftynine $(80 \%)$ of the 74 parents would choose sedation again and the same percentage of parents would recommend sedation for others.

Pain was the most consistent complaint; the percentage of patients reporting after 1 hour, 6 hours and 24 hours was 28\%, $21 \%$ and $14 \%$, respectively. Sleepiness and dizziness were the two next most common complaints at 1 hour after treatment (67\% and 57\% respectively).

Other complaints included vomiting, double vision, confusion, hallucination and tearfulness. Out of $26 \%$ of patients who had complaints, $12 \%$ had double vision and 8\% had nausea, as shown in Figure 3.

\section{Dentists' preference between general anaesthesia and intravenous sedation}

One hundred and one out of 221 questionnaires (45\%) were returned from dentists. Seventy of the dentists were male and 31 female. Fifty-eight dentists (56\%) preferred GA and 67 (66\%) preferred IV sedation.

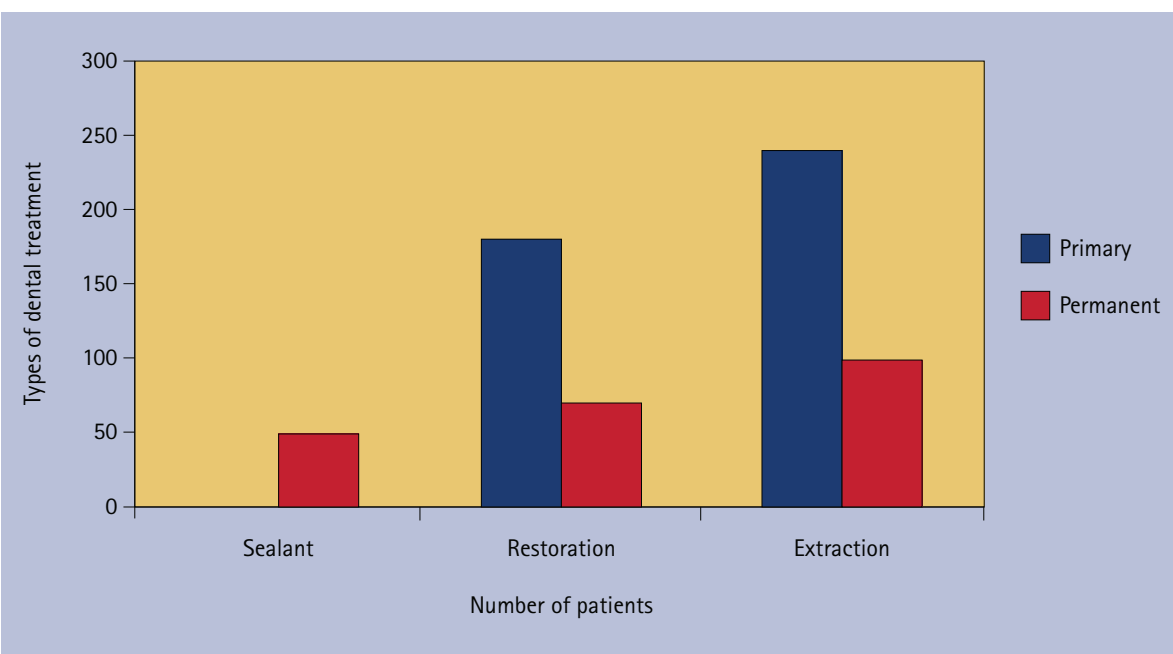

Fig. 1 Treatment provided under IV sedation

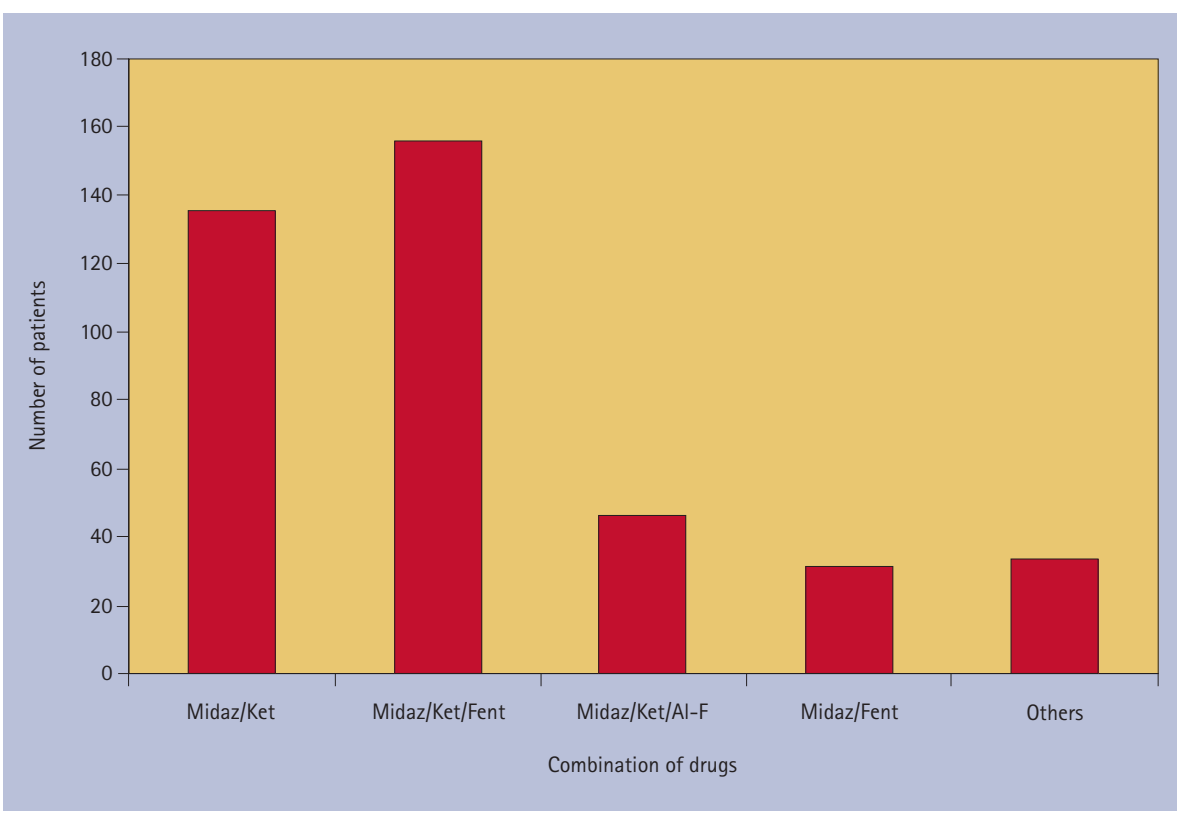

Fig. 2 Frequency of drug combinations used for sedation

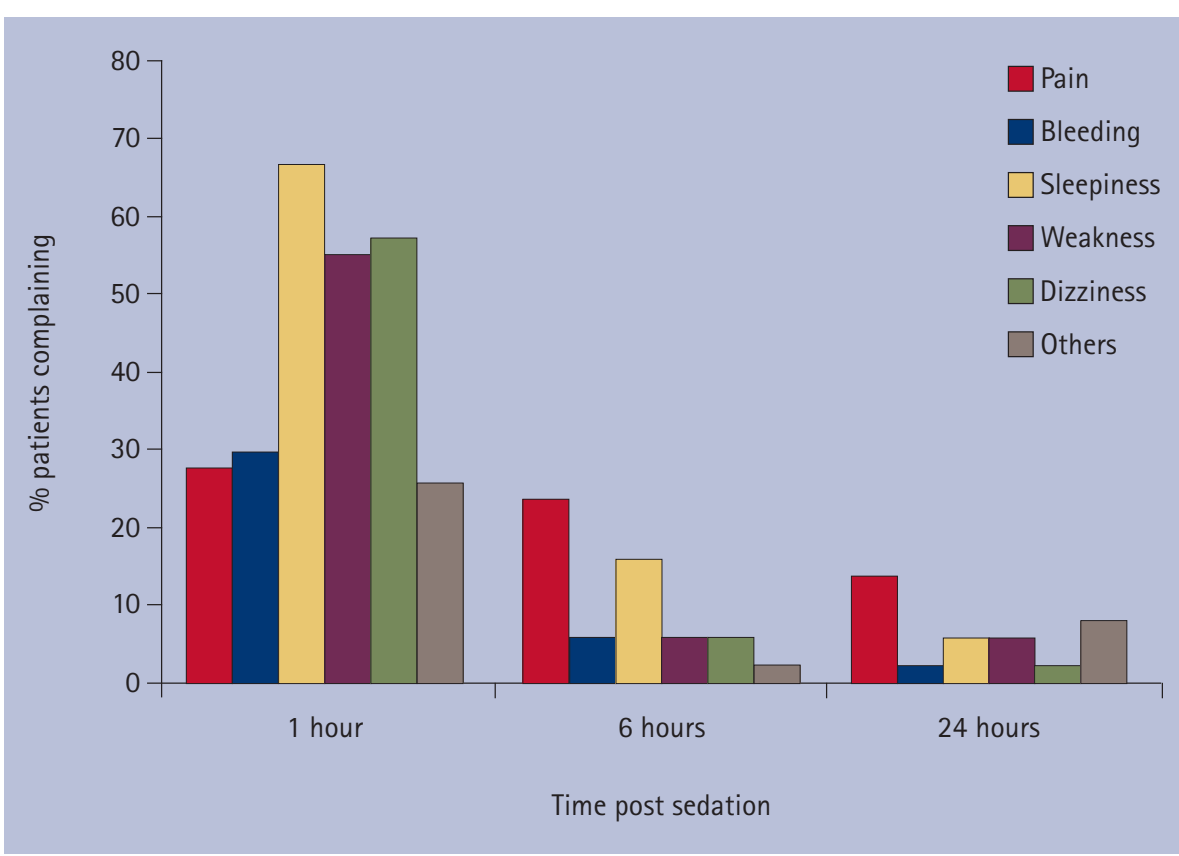

Fig. 3 Frequency of complaints 1 hour, 6 hours and 24 hours after sedation 
The main reasons for referring a child for GA included the patient being too young to co-operate (80\%), failing to respond to another form of behaviour management (79\%) and special need patients (56\%), while the least common reason was get treatment completed in one visit (15\%). The main reasons for referring a child for IV sedation were the patient failing to respond to another form of behaviour management (81\%), being too young to co-operate (75\%), and special need patient (49\%), while the least common reason was to get treatment completed in one visit (19\%).

The main ages for referring children for GA were between 2-6 years (58\%), 7-12 years (30\%) and over 12 years (7\%), while in IV sedation the main ages were between 2-6 years and 7-12 years (37\%).

Twenty-one (37\%) dentists referred one patient per month to have GA treatment, 25 (37\%) dentists referred one patient per month to have IV treatment, and 10 (15\%) dentists referred two patients a month for IV treatment.

Sixteen dentists (28\%) answered that the GA waiting time was between 1-3 months, 11 dentists (19\%) between 4-6 months, and 11 dentists (19\%) did not know, while for IV sedation 27 dentists (40\%) answered that the waiting time was less than 1 month, and 26 dentists (39\%) between 1-3 months.

When the dentists were asked if they recalled their patients after the GA or IV treatment, the answer was 'no' in 53\% and $55 \%$ of cases, respectively.

Almost half of the dentists (49\%) were very satisfied or satisfied with the GA service, while $17 \%$ were unsatisfied. Twentytwo dentists (61\%) were very satisfied or satisfied with the IV service and 11\% of dentists were unsatisfied.

\section{DISCUSSION}

\section{Audit of clinical practice of a dental sedation service in the primary care sector}

The majority of children were referred for caries management (78\%), and the rest for orthodontic extractions. This distribution was similar to that found in a previous study looking at referrals for dental work under general anaesthesia in the UK, where the main use of treatment was caries management. ${ }^{5}$ Most of the treatment (96\%) was completed in one visit; again this was reported in a previous study ${ }^{6}$ which showed that treatment was more likely to be completed in one visit with IV sedation when compared to other types of sedation. It is interesting to note that only half of the patients were given LA. This may be because of the type of procedure being provided; it could also, however, reflect the depth of the sedation.

A criticism often levelled at studies looking at the use of sedative techniques instead of GA is that these studies do not look at the long-term survival of the restorations placed. This study does not answer this question, nor was it designed to. Ultimately the quality of restorations placed should always be of the highest standard whether treatment is carried out under local anaesthesia, sedation or GA.

Sedation carried out in this study would best be described as 'alternative', with varying combinations of drugs being used. In general, evidence of the efficacy of drugs used for sedation is lacking, as identified by a recent systematic review, ${ }^{7}$ so it is difficult to pass comment as to the appropriateness or efficacy of the varying techniques used. All sedation was delivered by anaesthetists rather than non-medically qualified sedationists and no side-effects were reported by the anaesthetist or operator during the sedation or the recovery period. Obviously these findings do not rule out the risk of serious sequalae from administration of combinations of drugs, however in the patients studied this appeared to be a safe method of behaviour management.

For the audit of satisfaction, the authors suggested that 100 prospective questionnaires should be chosen; no sample calculation was carried out. The final response rate was $74 \%$ and can therefore be expected to give a reasonable representation of the patient satisfaction of this service. In this study patient satisfaction was high at $97 \%$, with $80 \%$ of patients stating they would choose IV sedation again. These results were similar to those reported previously. ${ }^{3}$

The systematic review of sedation for children in dentistry ${ }^{7}$ has shown that nausea (31\%) and sleep and drowsiness (44\%) are the most common adverse events reported. Sleepiness was the most common adverse event following sedation (56\%) in this study. Interestingly though, nausea did not seem to be a problem with only $8 \%$ of the patients reporting this symptom in our study.

\section{Dentists' preferences between general anaesthesia and intravenous sedation}

A convenience sample was selected for this part of the study as both PCTs involved were interested in sharing audit data. This also allowed investigation of both IV sedation and GA services as both were offered in these areas. However, this reduces the generalisability of this data to the UK as a whole. It is also important to note that dental extraction of non-impacted teeth under GA was considered to be a low priority procedure by these PCTs, meaning these procedures were not routinely funded in secondary care and if there were no specific risk factors, a special application for funds had to be made. This might have influenced dentists' referral patterns or perceptions of GA.

Unfortunately response rates were low so results have to be interpreted with caution. In general, results were broadly similar for both IV sedation and GA services. There were no obvious differences between GA and IV sedation with regard to dentist perception, however this data was not statistically tested due to the low response rate.

What should be concluded from this? Little data exist on current IV sedation services in the UK and this study goes some way to address this. It is clear that this clinic is providing a service that appears to be safe and well liked by patients. It is likely that if this service was not provided, some or all of these patients would not have been able to receive care in the primary sector. This service also appears to be viewed in a similar light by dentists referring to GA services. What this study cannot tell us is the relative safety, post-operative morbidity and cost of this approach when compared to GA. There is a clear need for clinical trials to directly compare sedation with GA for the provision of dental treatment.

\section{CONCLUSION}

Dental treatment for children was provided under IV sedation with most parents 
satisfied with the procedure. Little difference was seen between referring dentists' perceptions of IV sedation or GA.

1. Department of Health. A conscious decision. A review of the use of general anaesthesia and conscious sedation in primary dental care. London: Department of Health, 2000. http://www.dh.gov. uk/en/Publicationsandstatistics/Publications/ PublicationsPolicyAndGuidance/DH_4074702.
2. Strunin L. Intravenous conscious sedation for dental treatment: am I my brother's keeper? Anaesthesia 2007; 62: 645-647.

3. Wilson V, Girdler N M, Welbury R R. Randomised controlled cross over clinical trial comparing intravenous midazolam sedation with nitrous oxide sedation in children undergoing dental extraction. Br J Anaesth 2003; 91: 850-856.

4. Chadwick B L, Thompson S, Treasure E T. Sedation in Wales: a questionnaire. Br Dent J 2006; 201: 453-456.
5. Mason C, Holt R D, Rule D C. The changing pattern of day-care treatment for children in a London dental teaching hospital. Br Dent J 1995; 179: 136-140

6. Milnes A R. Intravenous procedural sedation: an alternative to general anaesthesia in the treatment of early childhood caries. J Can Dent Assoc 2003; 69: 298-302.

7. Matharu L, Ashley P F. Sedation of anxious children undergoing dental treatment. Cochrane Database Syst Rev 2006; (1): CD003877. 